\title{
Evaluation of Urine Cortisol and Psychological Factors in a Sample of Patients with Dental Anxiety
}

ISSN: 2637-7764

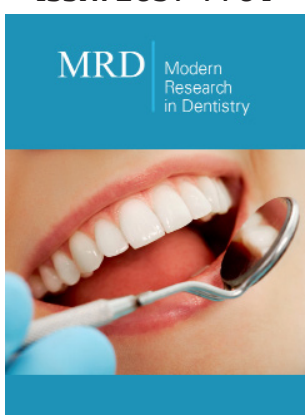

*1 Corresponding author: Chávez MMC, Pediatric Dentist, Venezuela

Submission: 眥June 25, 2018

Published: 㘹 March 13, 2019

Volume 3 - Issue 5

How to cite this article: Morales-Chávez MC, López-Labady J. Evaluation of Urine Cortisol and Psychological Factors in a Sample of Patients with Dental Anxiety. Mod Res Dent. 3(5). MRD.000571.2019. DOI: 10.31031/MRD.2019.03.000571

Copyright@ Smriti Jagdhari Golhar, This article is distributed under the terms of the Creative Commons Attribution 4.0 International License, which permits unrestricted use and redistribution provided that the original author and source are credited.

\author{
Morales-Chávez MC ${ }^{1 *}$ and López-Labady J² \\ ${ }^{1}$ Pediatric Dentist, Venezuela \\ ${ }^{2}$ Dentist, Venezuela
}

\begin{abstract}
High cortisol levels in all different body fluids are physiological markers of stress and anxiety. This might represent a useful tool to plan the dental management in children.

Objective: The aim of the present study was to determine the urinary cortisol values in children with signs of anxiety determined by the DASS scale before the dental evaluation.

Materials and Methods: A sample of 20 patients out of a total of 67, who attended the undergraduate clinic of the Faculty of Dentistry in Santa Maria University, Caracas, Venezuela; in a two-month period, were selected after they all answered the DASS scale and showed obvious signs of anxiety. Partial urine samples were taken from each patient with anxiety characteristics 10 minutes before the dentistry consultation. The samples were preserved in cold and then they were analyzed through chemiluminescence to determine cortisol serum levels. The data were analyzed by descriptive statistics.
\end{abstract}

Results: Urine mean cortisol level in anxious patients was $17.72 \mathrm{mcg} / \mathrm{dl}$.

Conclusion: The present study allowed us to conclude that urinary cortisol levels were raised by anxiety to a dental consultation in the pediatric population; as well as in the same way as children grow and progressively adapt to dental care and stress conditions; we were able to demonstrate how cortisol levels decrease. In relation to gender, girls show greater evidence of characteristics associated with stress compared to boys in similar conditions, which may be related to hormonal circumstances inherent to the gender itself as it has previously been found in the literature.

Keywords: Cortisol; Behavior management; Anxiety

\section{Introduction}

Dental anxiety can be described as an aversive emotional state of apprehension or worry in anticipation of a feared stimulus related to a dental treatment [1]. Dental fear and anxiety translate into complications in the management of pediatric patients. In fact, dental anxiety plays a major role in avoiding dental treatments; negatively affecting the oral health condition and causing an increase in the incidence [2] of cavities. Dental anxiety prevails worldwide and not limited to a specific population or country. This is a common problem that develops especially during childhood and adolescence. It has been reported that dental anxiety in children is influenced by many factors such as age, gender, education, socioeconomic status, ethnicity and culture, number of siblings, parental/maternal anxiety, previous dental and medical experiences among others [3]. Depending on the population and the method of measurement between $2.5-20 \%$ of people suffer high dental anxiety [4]. It must be recognized that the nature of the dental anxiety of a child can vary significantly and while some children exhibit fears or phobias in relation to specific dental stimulus such as needles, other children report generalized anxiety related with dental setting [5]. Each state of anxiety has different psychological changes with specific reactions in the body. The terms "dental anxiety" and "dental phobia" have been used in a similar way. Today, dental anxiety is distinguished from dental phobia. According to the international classification of diseases (World Health 
Organization ICD-10 Version 2015), dental phobia is a recognized disease that is accompanied by a disproportionate and sickly fear of objects and situations that leads to avoid behavior, which often results in cancellation of dental appointments [6].

In the same way, anxiety is usually defined as a physiological and psychological reaction generated by the organism as a defense mechanism against internal or external threats and has at least two components: One of the components is related with the hypothalamic-pituitary-adrenal (HPA) axis and the secretion of glycocorticoids such as cortisol in the blood stream. The second one related with the activation of the autonomic nervous system (ANS) and the release of catecholamines such as norepinephrine [7]. Cortisol levels are a very useful non-invasive biomarker to accurately measure stress levels, and therefore it has been used in different studies to evaluate anxiety in dentistry consultation [7-9]. There are different methods to determine the signs of anxiety, one of them is the DASS scale. The test consists of a list of 42 symptoms, each of which is going to be rated on a four point scale based on severity and frequency of each symptom during the last week. The DASS is a measure of mental health focusing on three traits: depression, anxiety and stress [10]. The aim of the present study was to determine the urinary cortisol level in children with signs of anxiety based on the DASS scale before the dental evaluation.

\section{Materials and Methods}

All the patients (67 children) who attended to undergraduate program unit in pediatric dentistry of Santa Maria University during the summer period were asked to answer the dental anxiety scale (DASS) to determine their psychological anxiety levels. Each of them were asked to use 4-point scale to rate the level of severity and frequency experienced in each state during the last week. Scores for Depression, Anxiety and Stress were calculated by summing the scores for the relevant items. Some features associated with Anxiety on DASS scale were: apprehension, panic, tremors dryness of the mouth, difficulty breathing, heart palpitations, sweating hands, concern about the procedure and possible loss of control. Twenty pediatric patients $(29,85 \%$ of total population) with ages between 4-10 years old; with a median of 8.15 years who showed some of these features were intentionally selected. Inclusion criteria: all the patients whose parents or guardians had signed an informed consent accepting to be part of the study and those who presented some of the anxiety symptoms included in DASS Scale, as well as all the patients with special needs, hormonal disorders or patients who by the time of the study were consuming any corticosteroids were excluded. The research was based on the ethical aspects established in the meeting of Helsinsky. Likewise, the ethical approval for the study was granted by the Bioethics Committee of the School of Dentistry of Santa Maria University. Partial urine samples were taken from each patient with anxiety features, 10 minutes before entering the dental consultation between 12:00 and 3:00pm to minimize the effect of the circadian rhythm in order to associate the anxiety level with cortisol levels. Samples were cryopreserved and sent to the lab in order to determine cortisol levels, and processed by chemiluminescence in an integrated system of immunoassay Abbott Architect ci 16200. The results were evaluated statistically with SPSS software version 20.0 IBM 2015 with a descriptive analysis.

\section{Results}

Sixty-seven (67) patients answered DASS Scale. Of these, Twenty (20) children with a median age of 8.15 years and obvious anxiety features according to DASS scale participated in this study. Thirteen (13) patients (65\%) were girls and Eight (8) (35\%) were boys. When the urine cortisol level was assessed in patients with anxiety, a median of $17.72 \mathrm{mcg} / \mathrm{dl}$ was observed, representing thirteen points above the reference value $(4 \pm 1.5 \mathrm{mcg} / \mathrm{dl}) .55 \%$ of the patients showed altered values that oscillated between 17.9 and $34.1 \mathrm{mcg} / \mathrm{dl}$. Those results represent an important correlation between DAS scores and urine cortisol levels. In relation to age, the group of children with ages between 4 and 6 years old had higher cortisol levels than older children. When we related results with gender, it was determined that cortisol levels were higher in female patients $25.82 \pm 3.15 \mathrm{mcg} / \mathrm{dl}$ and lower in male patients $18.35 \pm 4.77 \mathrm{mcg} / \mathrm{dl}$.

\section{Discussion}

In the present, the study of the physiological behavior regarding anxiety has become an important need, in order to give a real answer vis-à-vis to these circumstances often presented by patients who attend the dental consultation, mainly pediatric patients. These findings provide additional support for the validity and use of DAS as a tool for estimating the level of dental anxiety perceived by the patients and it is well related to the level of stress measured by physiological analysis of cortisol level in saliva. That indicates that both tools can be used as a method for the assessment of dental anxiety [10]. 55\% of the patients with anxiety according to DASS scale showed altered urine cortisol levels. Zwain et al. [11] also observed a significantly higher increase in the levels of cortisol in patients who showed anxiety criteria according to DASS scale. This shows that this survey is effective to determine the presence of anxiety symptoms and it can be very helpful when planning dental treatment. Cortisol is an adrenocortical hormone that diffuses to all body fluids and represents today an investigation target, since high levels in serum; urine and saliva have been observed under stress conditions [2]. Studies reveal that the chronic persistence of this hormone has as consequences the delay and alteration of wound healing and local suppression of the immune system [12,13]. The results of this study show how urine cortisol levels before dental treatment were significantly higher in most children with obvious signs of anxiety, such as crying apprehensive and difficulty breathing. Studies such as Furlan et al. [7] also evidence that salivary cortisol levels are significantly higher before a dental consultation without detecting signs of stress in the behavior of children, while Gomes et al. [14] observed lower salivary levels before consultation, than during a simple treatment such as dental prophylaxis. In relation to age, the group of children with ages between 4 and 6 years old had higher cortisol levels than older children, which is consistent with the literature that establishes that younger children tend to be more anxious in the dental office compared to older children [3]. 
This study revealed that urine cortisol levels before dentistry consultation are higher in girls. Similar results were observed by Armfield et al. [15] who concluded that a higher percentage of females than males reported greater fear. Other evidences about the difference in dental anxiety between boys and girls have been inconsistent. Most investigators reported higher levels of dental anxiety in girls [16-18]. The characteristics observed in this research allowed us to assume that the signs of anxiety in children are directly related to high levels of urine cortisol. This can lead us to decide the most convenient dental procedures for pediatric patients with evident stress and anxiety markers, mainly the surgical procedures, due to possible immune alterations and wound healing delayed. Even though the most recent studies use salivary cortisol measurements; because that represents a simple and minimally invasive method for patients, it is too difficult for both, parents and professionals, to schedule a dentistry appointment for the child based on the levels of this hormone by using this method.

The sample must be taken by the dentist; therefore, assigning parents this complex task could be problematic because sample collection implies some elements to consider, such as circadian rhythm and food intake. Parents and guardians are familiar with urine sampling, and this has been the traditional procedure to measure cortisol. The answer to which could be the ideal moment for a pediatric patient to attend dentistry consultation has remained an issue for years, especially in those cases where oral condition is very poor and complex dental procedures are necessary with successive visits in order to re-establish oral health, which implies significant stress and anxiety levels, and a significant increase in serum, urine and saliva cortisol levels. If we consider that high cortisol levels involve psychological and physiological damage in these patients, we could have a mechanism that allow us to plan appointments and procedures to be performed in alliance with parents depending on urine cortisol levels; using a simple and minimally invasive sample collection method for them.

\section{Conclusion}

The present study allows us to conclude that urine cortisol levels were elevated due to the anxiety caused by the dental consultation in the pediatric population. In the same way we were able to demonstrate that as children grow and progressively adapt to oral care and stress conditions, cortisol levels decrease. In relation to gender, girls show more evidence compared to boys in similar conditions, which may be related to hormonal circumstances inherent to the gender itself as it has previously been found in the literature.

\section{References}

1. Armfield JM (2010) How do we measure dental fear and what are we measuring anyway? Oral Health Prev Dent 8(2): 107-115.
2. Jafarzadeh M, Arman S, Pour F (2013) Effect of aromatherapy with orange essential oil on salivary cortisol and pulse rate in children during dental treatment: A randomized controlled clinical trial. Adv Biomed Res 2: 10 .

3. Alasmari AA, Aldossari GS, Aldossari MS (2018) Dental anxiety in children: A review of the contributing factors. J Clin Diagn Res 12(4): 1-3.

4. Saatchi M, Abtahi M, Mohammadi G, Mirdamadi M, Binandeh ES (2015) The prevalence of dental anxiety and fear in patients referred to Isfahan Dental School. Dent Res J (Isfahan) 12(3): 248-253.

5. Porritt J, Buchanan H, Hall M, Gilchrist F, Marshman Z (2013) Assessing children's dental anxiety: A systematic review of current measures. Community Dent Oral Epidemiol 41(2): 130-142.

6. Naumova EA, Faber S, Lindner P, Wannemueller A, Sandulescu T, et al. (2016) Parallel study about the effects of psychotherapy on patients with dental phobia determined by anxiety scores and saliva secretion and composition. BMC Oral Health 17(1): 32.

7. Furlan NF, Gavião MB, Barbosa TS, Nicolau J, Castelo PM (2012) Salivary cortisol, alpha-amylase and heart rate variation in response to dental treatment in children. J Clin Pediatr Dent 37(1): 83-87.

8. Pani SC, Al Askar AM, Al Mohrij SI, Al Ohali TA (2011) Evaluation of stress in final-year Saudi dental students using salivary cortisol as a biomarker. J Dent Educ 75(3): 377-384.

9. Blomqvist M, Holmberg K, Lindblad F, Fernell E, Ek U, et al. (2007) Salivary cortisol levels and dental anxiety in children with attention deficit hyperactivity disorder. Eur J Oral Sci 115(1): 1-6.

10. Brown TA, Chorpita BF, Korotitsch W, Barlow DH (1997) Psychometric properties of the Depression Anxiety Stress Scales (DASS) in clinical samples. Behav Res Ther 35(1): 79-89.

11. Zwain AMH, Salih BA (2014) Assessment of dental anxiety by physiological measurement (salivary cortisol) and psychological measurement (dental anxiety scale) in children. J Babylon Univers Pure Applied Sc 22(9): 2568-2578.

12. Ebrecht M, Hextall J, Kirtley LG, Taylor A, Dyson M, et al. (2004) Perceived stress cortisol levels and predict speed of wound healing in healthy male adults. Psychoneuroendocrinology 29(6): 798-809.

13. Walburn J, Vedhara K, Hankins M, Rixon L, Weinman J (2009) Psychological stress and wound healing in humans: A systematic review and meta-analysis. J Psychosom Res 67(3): 253-271.

14. Gomes HS, Vieira LA, Costa PS, Batista AC, Costa LR (2016) Professional dental prophylaxis increases salivary cortisol in children with dental behavioural management problems: a longitudinal study. BMC Oral Health 16(1): 74.

15. Armfield JM, Spencer AJ, Stewart JF (2006) Dental fear in Australia: who's afraid of the dentist? Aust Dent J 51(1): 78-85.

16. Kakkar M, Wahi A, Thakkar R, Vohra I, Shukla AK (2016) Prevalence of dental anxiety in 10-14 years old children and its implications. J Dent Anesth Pain Med 16(3): 199-202.

17. Yildirim TT, Dundar S, Bozoglan A, Karaman T, Dildes N, et al. (2017) Is there a relation between dental anxiety, fear and general psychological status? PeerJ 5: e2978.

18. El Housseiny AA, Merdad LA, Alamoudi NM, Farsi NM (2015) Effect of child and parent characteristics on child dental fear ratings: analysis of short and full versions of children's fear survey schedule-dental subscale. Oral Health Dent Manag 14(1): 9-16. 